\title{
Validación de un cuestionario breve para medir conductas alimentarias de riesgo
}

\author{
Claudia Unikel-Santoncini, D ra en Psic, ${ }^{(1)}$ letza Bojórquez-Chapela, MC, ${ }^{(2)}$ \\ Silvia Carreño-García, Lic en Psic. ${ }^{(1)}$
}

\author{
Unikel-Santoncini C, Bojórquez-Chapela I, \\ Carreño-García S. \\ Validación de un cuestionario breve \\ para medir conductas alimentarias de riesgo. \\ Salud Publica Mex 2004;46:509-515. \\ disponible en: http://www.insp.mx/salud/46/eng
} El texto completo en inglés de este artículo está

\begin{abstract}
Resumen
Objetivo. Mostrar los resultados de confiabilidad y validez de un cuestionario para identificar conductas alimentarias de riesgo. Material y métodos. El cuestionario se aplicó a una muestra de mujeres con diagnóstico de trastorno alimentario, en tratamiento en la Clínica de Trastornos de la ConductaA limentaria, del Instituto $\mathrm{N}$ acional de Psiquiatría, en el periodo septiembre-diciembre de 2002, y a una muestra de mujeres estudiantes de nivel medio y medio superior en la Ciudad de México, en octubre del mismo año. Se hizo análisis de consistencia interna (alfa de Cronbach) y análisis factorial de componentes principales con rotación oblicua; mediante tablas de $2 \times 2$ se determinaron el punto de corte, la sensibilidad, la especificidad y los valores predictivos del cuestionario. Resultados. El instrumento tiene una alta confiabilidad $(\alpha=0.83)$ y una estructura interna de tres factores con una varianza explicada de $64.7 \%$. El análisis discriminante mostró que casi $90 \%$ de los casos fueron correctamente agrupados. Conclusiones. El instrumento presentado es una opción confiable y válida para la evaluación de conductas alimentarias de riesgo en la población de las muestras estudiadas. El texto completo en inglés de este artículo también está disponible en: http://www.insp.mx/salud/46/eng
\end{abstract}

Palabras clave: conducta alimentaria de riesgo; reproducibilidad de resultados; México

\author{
Unikel-Santoncini C, Bojórquez-Chapela I, \\ Carreño-García S. \\ Validation of a brief questionnaire \\ to measure the risk of abnormal eating behaviors. \\ Salud Publica Mex 2004;46:509-515. \\ The English version of this paper \\ is available at: http://www.insp. $\mathrm{mx} / \mathrm{salud} / 46 / \mathrm{eng}$
}

\begin{abstract}
A bstract
Objective To assess the validity and reliability of a questionnaire for the screening of risk eating behaviors. Material and Methods. The questio nnaire was applied to female high school students in Mexico City in October2002, as well as to a sample of eating disorder patients seen at the Eating D isorders U nit of the N ational Institute of Psychiatry between September and December 2002. Statistical methods included internal consistency analysis (C ronbach's alpha) and factor and principal component analysis with oblique rotation. The cutoff point, sensitivity, specificity, and predictive values of the questionnaire were determined using $2 \times 2$ tables. Results. The questionnaire showed a high reliability $(\cdot=0.83)$ and a three-factor structure with $64.7 \%$ of the total explained variance.A discriminant analysis showed that almost $90 \%$ of cases were correctly classified. Conclusions. This questionnaire is reliable and valid for assessing risk eating behaviors in the study population. The English version of this paper is available at: http:// www.insp.mx/salud/46/eng
\end{abstract}

Key words: risky feeding behavior; reproducibility of results; Mexico

(1) Dirección de Investigaciones Epidemiológicas y Psicosociales, Instituto N acional de Psiquiatría Ramón de la Fuente Muñiz. México, DF, México.

(2) Facultad de Medicina, Universidad N acional Autónoma de México, México, DF, México.

Fecha de recibido: 30 de marzo de 2003 - Fecha de aprobado: 6 de julio de 2004

Solicitud de sobretiros: D ra. Claudia U nikel Santoncini. Dirección de Investigaciones Epidemiológicas y Psicosociales, Instituto N acional de Psiquiatría Ramón de la Fuente Muñiz. Calzada X ochimilco 101, Huipulco, Tlalpan 14370 México, DF. Correo electrónico: unikels@ imp.edu.mx 
os trastornos de la conducta alimentaria (TCA) son un problema de salud mental cada vez más frecuente entre las mujeres jóvenes. ${ }^{1,2} \mathrm{Si}$ bien los trastornos definidos por el Manual Diagnóstico y Estadístico de las Enfermedades Mentales (DSM-IV, por sus siglas en inglés), anorexia nervosa, bulimia nervosa, y trastorno de la conducta alimentaria no especificado tienen prevalencias de apenas $0.5 \mathrm{a} 3 \%,{ }^{3}$ las conductas alimentarias de riesgo son mucho más frecuentes. No existe un concepto unificado de las conductas de riesgo, pero este término, así como los de síndromes parciales, conductas alimentarias anormales y trastornos subclínicos hacen referencia a todas aquellas manifestaciones similares en forma, pero menores en frecuencia e intensidad a las de los TCA. ${ }^{4}$ En México se han encontrado porcentajes desde 5 hasta $18 \%$ de conductas alimentarias de riesgo en muestras de mujeres estudiantes universitarias, ${ }^{5}$ adolescentes ${ }^{6}{ }^{6}$ estudiantes de bachillerato de la Ciudad de México. ${ }^{7}$

Los estudios de seguimiento por periodos de entre 10 y 15 años de grupos de pacientes con anorexia nervosa han mostrado que los síntomas persisten en 12 a $14 \%$ de los $\operatorname{casos}^{8}$ y las tasas de recaída son elevadas, entre 4 y $27 \%,{ }^{9,10}$ por lo que puede hablarse de un padecimiento crónico. Según el estudio llevado a cabo por Sullivan, ${ }^{11}$ la tasa de mortalidad agregada asociada con la anorexia nervosa es 12 veces mayor que la tasa anual de mortalidad debida a todas las causas para mujeres de entre 15 a 24 años de edad en la población general. Los TCA se asocian con gran número de complicaciones fisiológicas en la adultez temprana, ${ }^{12}$ además de presentarse con un porcentaje elevado de comorbilidad psiquiátrica, ${ }^{13,14}$ principalmente con trastornos afectivos. ${ }^{15,16}$

Lo mencionado anteriormente, aunado a que la población más afectada con los TCA se encuentra en una etapa crucial de su desarrollo físico, mental y social, convierte a la detección temprana de casos en una tarea por demás importante. Esto es igualmente cierto en cuanto a los síndromes parciales, que podrían ser incluso más preocupantes porque, al mantenerse el sujeto afectado dentro de los límites de lo considerado normal, no es detectado por profesionales médicos, educadores o por sus familiares y, al no ser considerado un problema, no recibe tratamiento.

Dado el carácter de problema de salud mental pública de los TCA es importante contar con instrumentos adecuados para su detección, con fines tanto clínicos como epidemiológicos. En México, por razones principalmente de recursos, son pocas las encuestas que logran tener una verdadera representatividad nacional. Siendo los TCA una enfermedad poco reconocida como problema de salud pública, es improbable que se consi- ga apoyo para una encuesta amplia que se ocupe exclusivamente de ellos. En cambio, es factible incluir unas cuantas preguntas al respecto en el contexto de encuestas más amplias de salud mental.

En la investigación acerca de los TCA se han empleado diferentes cuestionarios autoaplicables, de los cuales los más utilizados son el Eating Attitudes Test $(\text { EAT })^{17}$ y el Eating Disorders Inventory (EDI), ${ }^{18}$ que han sido validados para población mexicana por Alvarez, Mancilla, Vásquez, Unikel, Caballero y Mercado, ${ }^{19} \mathrm{Al}-$ varez ${ }^{20}$ y Alvarez y Franco. ${ }^{21}$ A pesar de la elevada confiabilidad y validez de ambos instrumentos, la principal desventaja que presentan es su extensión. En el caso del EDI existe, además, el riesgo de que las preguntas sobre características psicológicas (conciencia interoceptiva, desconfianza interpersonal, ineficacia, miedo a madurar y perfeccionismo) resulten confusas en su significado al ser aplicadas a poblaciones culturalmente diversas. ${ }^{22}$ Por ejemplo, en una población semiurbana del estado de Michoacán, ${ }^{23}$ las subescalas del EDI que miden actitudes y conductas alimentarias obtuvieron correlaciones ítem-total y confiabilidad más adecuadas que las preguntas sobre características psicológicas.

En un intento por solucionar estos problemas, y de obtener datos representativos acerca de la frecuencia de conductas alimentarias de riesgo en una población abierta, en 1997 se diseñó un instrumento de 11 reactivos para la detección de conductas alimentarias de riesgo. Estos 11 reactivos se incluyeron por primera vez en la Encuesta sobre la Prevalencia del Consumo de Drogas y Alcohol en la Población Estudiantil del Distrito Federal, en 1997, obteniéndose con ello información estadísticamente confiable sobre conductas alimentarias de riesgo en esta población. ${ }^{7}$ Dicho instrumento establece preguntas principalmente por conductas, y la frecuencia con la que éstas se manifiestan, con lo cual permite obtener datos sobre prácticas dañinas sin importar con qué elementos psicológicos se relacionen. El objetivo del presente trabajo es mostrar los resultados de confiabilidad y validez de ese cuestionario, así como la sensibilidad y especificidad del mismo.

\section{Material y métodos}

\section{Sujetos}

El cuestionario autoaplicable se probó en una muestra de 78 pacientes mujeres con diagnóstico de trastorno de la conducta alimentaria, de acuerdo con los criterios del DSM-IV, ${ }^{3}$ que acudieron por primera vez a la Clínica de Trastornos de la Conducta Alimentaria del Instituto Nacional de Psiquiatría Ramón de la Fuente Muñiz, de México (INP), en el periodo septiembre-diciem- 
brede 2002. La distribución de la muestra por diagnóstico fue: $48.7 \%$ con bulimia nervosa, $9 \%$ con anorexia nervosa restrictiva, $5.1 \%$ con anorexia nervosa compulsivo-purgativa, y $37.3 \%$ con un TCA no especificado. La edad promedio fue $20.2 \pm 4.3$ años, con un índice de masa corporal (IMC) promedio de $21.2 \pm 5.2$. El $30 \%$ de los padres y $20 \%$ de las madres reportaron una escolaridad de preparatoria o mayor.

El grupo de comparación fue una muestra de estudiantes de nivel medio y medio superior de escuelas públicas y privadas de la Ciudad de México $(n=468)^{24,25}$ con una edad promedio de $19.3 \pm 3.9$ y un IMC promedio de $21.6 \pm 3.4$. El $60 \%$ de los padres y $45 \%$ de las madres informaron estudios de preparatoria o mayores.

\section{Instrumento}

El cuestionario fue elaborado con base en los criterios diagnósticos del DSM-IV. ${ }^{7}$ Consta de 11 preguntas sobre preocupación por engordar, práctica de atracones, sensación de falta de control al comer y conductas alimentarias de tipo restrictivo (dietas, ayunos, ejercicio y uso de pastillas para bajar de peso) y purgativo (vómito autoinducido, uso de laxantes y de diuréticos), en los tres meses previos a la aplicación del instrumento. La versión original contaba con tres opciones de respuesta; sin embargo, se agregó una opción más para incrementar la variabilidad en las respuestas de los sujetos: nunca o casi nunca, algunas veces, frecuentemente (dos veces en una semana) o muy frecuentemente (más de dos veces en una semana), y la mayor puntuación en el cuestionario corresponde a mayor cantidad de anomalías en la conducta alimentaria (ver anexo).

Se aplicaron, además, las escalas de sintomatología depresiva ${ }^{26}$ e ideación suicida ${ }^{27}$ del Centro de Estudios Epidemiológicos -Center of Epidemiological Studies of Depression, CES-D-, en sus versiones validadas en adolescentes mexicanos, ${ }^{28,29}$ ya que de acuerdo con la literatura son fenómenos que tienen una comorbilidad elevada con trastornos afectivos, de tal manera que se esperaría una asociación positiva con la presencia de conductas alimentarias de riesgo. . $^{15,30,31}$

\section{A nálisis de datos}

Con el objetivo de conocer las características psicométricas del cuestionario se llevaron a cabo análisis de consistencia interna (alfa de Cronbach) y análisis factorial de componentes principales con rotación oblicua con la totalidad de la muestra comunitaria $(n=468)$. Se utilizó la muestra clínica $(n=78)$ y una submuestra aleatoria obtenida a partir de la muestra comunitaria $(n=93)$ para realizar el análisis de discriminantes paso a paso tipo
Mahal. Posteriormente, se determinaron el punto de corte del cuestionario, los valores de sensibilidad, especificidad y valores predictivos, mediante el método de tablas de $2 \times 2,32$ y finalmente, para determinar la validez concurrente del mismo, se hizo un análisis de correlación del cuestionario de conducta alimentaria de riesgo con la escala de Depresión del Centro de Estudios Epidemiológicos (CES-D) ${ }^{26}$ y la escala de ideación suicida, ${ }^{27}$ utilizando la totalidad de sujetos de ambas muestras. Los datos fueron capturados en computadora y analizados con el programa SPSS para Windows, versión 10.0 .

\section{Resultados}

Se desarrolló el análisis de consistencia interna de la escala, considerando como válidos los valores de las correlaciones ítem-total iguales o mayores a 0.30. La pregunta sobre uso de enemas, supositorios y lavativas obtuvo una puntuación menor a 0.30 , por lo que se decidió eliminarla para hacer los análisis posteriores. La escala en su conjunto obtuvo un valor de consistencia interna alfa de Cronbach de 0.83 .

A continuación se realizó un análisis factorial de componentes principales con rotación oblicua. Este arrojó una distribución en tres factores puros que explicaron $64.7 \%$ de la varianza total en siete iteraciones. El factor 1 agrupó los ítems: práctica de atracones, sensación de falta de control al comer, vómito autoinducido y práctica de ayunos, y explicó $42.3 \%$ de la varianza total $(\alpha=0.74)$. El factor 2 agrupó los ítems: uso de pastillas, uso de diuréticos y uso de laxantes para bajar de peso, y explicó $12.0 \%$ de la varianza total $(\alpha=0.72)$. El factor 3 agrupó los ítems: "me preocupa engordar", práctica de dietas y práctica de ejercicio para bajar de peso, y explicó $10.2 \%$ de la varianza total $(\alpha=0.76)$ (cuadro I).

El siguiente paso fue obtener la validez predictiva mediante un análisis de discriminantes. La matriz de estructura arrojó una sola función en tres iteraciones formada por tres ítems: autoinducción del vómito, práctica de ayunos y práctica de dietas, con cargas discriminantes de $0.52,0.76$ y 0.80 , respectivamente. La función obtuvo un valor Eigen de 1.64, 100\% de la varianza explicada y una correlación canónica de 0.78 ; es decir, sólo $22 \%$ de la varianza no fue explicada por el modelo $(1-0.78=0.22)$. Los resultados señalan que las variables incluidas en el análisis correlacionan adecuadamente y la función canónica discriminante es significativa al 0.001. Como último paso en el análisis se obtuvo el resumen de clasificación, en el que se pudo observar que $88.8 \%$ de los casos fueron agrupados correctamente (cuadro II). 


\section{Cuadro I \\ Resultado del anÁlisis factorial del CUestionario BREVE PARA MEDIR CONDUCTAS ALIMENTARIAS DE RIESGO. Ciudad de México, 2002.}

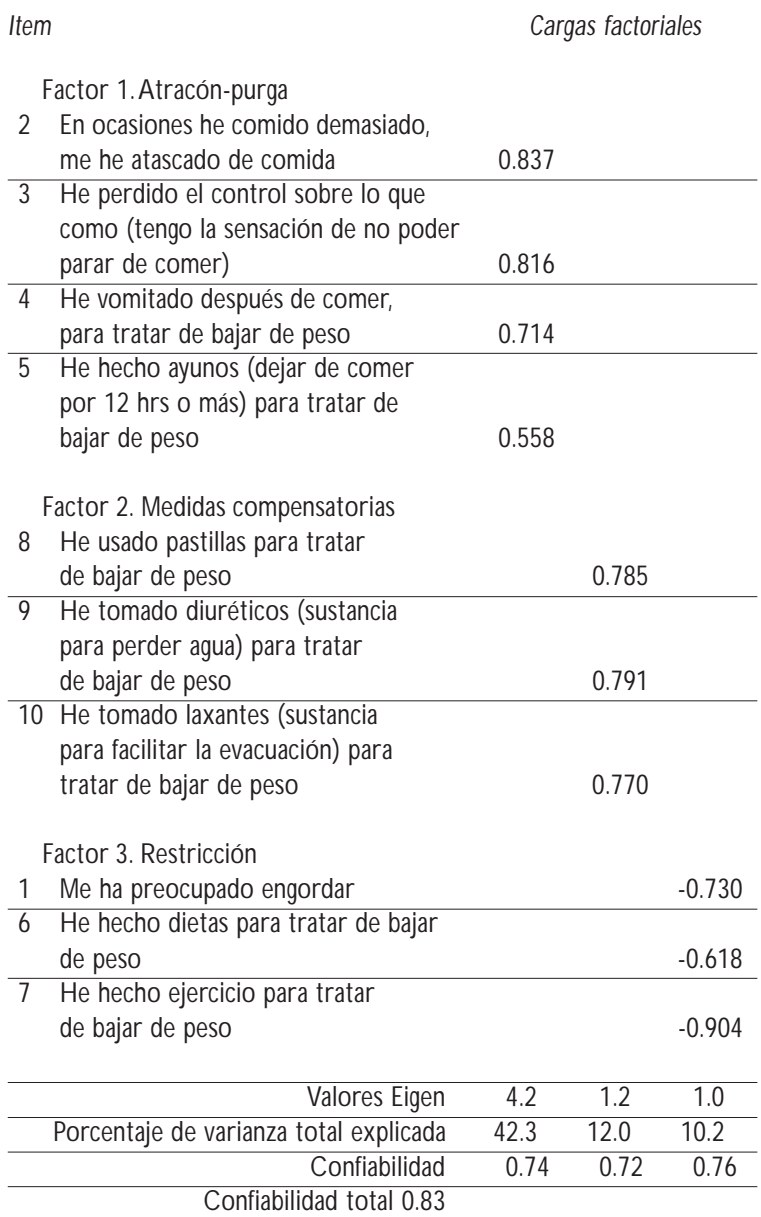

En seguida se determinó el punto de corte de la escala, o el punto crítico que delimita la frontera entre el grupo funcional y el disfuncional. Se obtuvieron la sensibilidad y especificidad de siete diferentes puntuaciones del cuestionario, ${ }^{7-13}$ elegidas con base en las puntuaciones medias totales obtenidas por el grupo comunitario. A partir de los análisis el punto de corte de 10 fue el que mostró los mejores resultados: arrojó una sensibilidad de 0.81 y una especificidad de 0.78 , con un valor predictivo positivo de 0.38 y un valor predictivo negativo de 0.96 (cuadro III). Debido a que el valor predictivo positivo se calculó con una prevalencia obtenida de esta misma muestra se hizo un análisis complementario utilizando una prevalencia $(16 \%)$ obtenida de una muestra representativa de estudian-

\section{Cuadro II \\ Validez predictiva del CUestionario breVe \\ PARA MEDIR CONDUCTAS ALIMENTARIAS DE RIESGO MEDIANTE ANÁLISIS DE DISCRIMINANTES. Resumen de Clasificación. Ciudad de México, 2002.}

\begin{tabular}{lccc} 
& \multicolumn{2}{c}{ Membresía de grupo predicha } & \\
\cline { 2 - 3 } Grupo original & $\begin{array}{c}\text { Comunitario } \\
n\end{array}$ & $\begin{array}{c}\text { Clínico } \\
n\end{array}$ & Total \\
Comunitario & 88 & 5 & 93 \\
\hline Clínico & 14 & 64 & 78 \\
& $\%$ & $\%$ & $\%$ \\
Comunitario & 94.6 & 5.4 & 100.0 \\
\hline Clínico & 17.9 & 82.1 & 100.0
\end{tabular}

Total de casos correctamente clasificados $=88.8 \%$

tes de sexo femenino con edad similar (entre 18 y 19 años) del Distrito Federal; ${ }^{7}$ como se puede observar, las variaciones en el resultado fueron mínimas (cuadro III).

Para obtener la validez concurrente del cuestionario se llevaron a cabo correlaciones momento-producto de Pearson entre los valores medios obtenidos para la puntuación total del cuestionario de conductas alimentarias y cada uno de los factores resultantes, con las puntuaciones totales de la escala de sintomatología depresiva CES-D y la escala de ideación suicida (cuadro IV). Como se observa, todas las correlaciones fueron positivas y significativas al 0.01 . Las correlaciones mayores a 0.50 se obtuvieron entre la puntuación total del cuestionario de conductas alimentarias de riesgo con el CES-D (0.543), con la escala de ideación suicida (0.501) y con la subescala atracón-purga (0.509), mientras que el resto fluctuó en un rango de 0.484 a 0.314 (cuadro IV).

\section{Discusión}

Los datos presentados muestran que el cuestionario de conductas alimentarias de riesgo es capaz de diferenciar entre una población clínica y una comunitaria, y tiene valores de confiabilidad (0.83) y validez apropiados para las muestras estudiadas.

Los valores obtenidos en el análisis de correlación con las escalas de sintomatología depresiva e ideación suicida brindan información importante respecto de la validez concurrente del cuestionario, al coincidir con los datos reportados en la literatura acerca de la comorbilidad de los TCA con trastornos afectivos ${ }^{13,14,16}$ y la mortalidad asociada con suicidio. ${ }^{11,15,30,31}$ Asimismo, se 


\section{Cuadro III \\ Datos de SENSIBILIDAD Y ESPECIFICIDAD DEL CUESTIONARIO BREVE PARA MEDIR CONDUCTAS ALIMENTARIAS DE RIESGO. Ciudad de México, 2002.}

\begin{tabular}{|c|c|c|c|c|c|c|c|c|}
\hline \multirow[b]{2}{*}{ Punto de corte } & \multirow[b]{2}{*}{ Sensibilidad } & \multirow[b]{2}{*}{ Especificidad } & \multirow[b]{2}{*}{$\begin{array}{c}\text { Falsos } \\
\text { positivo } \\
\%\end{array}$} & \multirow[b]{2}{*}{$\begin{array}{c}\text { Falsos } \\
\text { negativo } \\
\%\end{array}$} & \multicolumn{2}{|c|}{ Prevalencia $=0.14$} & \multicolumn{2}{|c|}{ Prevalencia $=0.16$} \\
\hline & & & & & $\begin{array}{l}\text { Valor predictivo } \\
\text { positivo }\end{array}$ & $\begin{array}{c}\text { Valor predictivo } \\
\text { negativo }\end{array}$ & $\begin{array}{l}\text { Valor predictivo } \\
\text { positivo }\end{array}$ & $\begin{array}{l}\text { Valor predictivo } \\
\text { negativo }\end{array}$ \\
\hline 7 & 0.91 & 0.65 & 35 & 9 & 0.30 & 0.98 & 0.33 & 0.79 \\
\hline 8 & 0.90 & 0.72 & 28 & 10 & 0.34 & 0.98 & 0.37 & 0.97 \\
\hline 9 & 0.87 & 0.74 & 26 & 13 & 0.36 & 0.97 & 0.38 & 0.96 \\
\hline 10 & 0.81 & 0.78 & 22 & 19 & 0.38 & 0.96 & 0.41 & 0.95 \\
\hline 11 & 0.77 & 0.83 & 17 & 23 & 0.43 & 0.96 & 0.46 & 0.95 \\
\hline 12 & 0.69 & 0.86 & 14 & 31 & 0.45 & 0.94 & 0.50 & 0.93 \\
\hline 13 & 0.59 & 0.90 & 10 & 41 & 0.48 & 0.93 & 0.52 & 0.92 \\
\hline
\end{tabular}

Cuadro IV

VALIDEZ CONCURRENTE del CUESTIONARIO BREVE PARA MEDIR CONDUCTAS ALIMENTARIAS DE RIESGO, mediante matriz de CorRelaciones. Ciudad de México, 2002.

\begin{tabular}{|c|c|}
\hline $\begin{array}{l}\text { Conductas } \\
\text { alimentarias de riesgo }\end{array}$ & $\begin{array}{c}\text { Factor } 1 . \\
\text { Atracón-purga }\end{array}$ \\
\hline
\end{tabular}

Conductas alimentarias de riesgo

\begin{tabular}{|c|c|c|c|c|c|}
\hline Atracón-purga & $0.837^{*}$ & - & & & \\
\hline \multicolumn{6}{|l|}{ Factor 2.} \\
\hline Medidas compensatorias & $0.716 *$ & $0.477^{*}$ & - & & \\
\hline \multicolumn{6}{|l|}{ Factor 3.} \\
\hline Restricción & $0.901 *$ & $0.594 *$ & $0.505 *$ & - & \\
\hline CES-D & $0.543^{*}$ & $0.509 *$ & $0.318^{*}$ & $0.484^{*}$ & - \\
\hline Ideación suicida & $0.501 *$ & $0.484^{*}$ & $0.314 *$ & $0.427 *$ & $0.752 *$ \\
\hline
\end{tabular}

${ }^{*} p<0.01$

CES-D: Center of Epidemiological Studies of Depression

observa una validez predictiva aceptable que permite clasificar correctamente una elevada proporción de los casos (88.8\%).

Considerando que los TCA son un padecimiento de baja prevalencia, y puesto que el principal objetivo de un instrumento de tamizaje es la detección de posibles casos clínicos y subclínicos, se buscó un punto de corte con alta sensibilidad. De acuerdo con Altman y Bland, ${ }^{32,33}$ a medida que una enfermedad presenta una menor prevalencia, como es el caso de los TCA, es más probable que un resultado negativo muestre la presencia de normalidad, y menos probable que un resultado positivo realmente indique anormalidad; es decir, que de acuerdo con los resultados obtenidos, de cada 100 personas que obtengan una puntuación menor al punto de corte, 96 serán correctamente clasificadas como no casos y cuatro como falsos negativo, pero de cada 100 personas que puntúen por arriba del punto de corte, sólo 38 estarán correctamente clasificadas como verdaderos positivo, o 41 al utilizar la prevalencia obtenida de la muestra representativa de población estudiantil femenina.

Las conductas alimentarias patológicas se presentan a lo largo de un continuo, en el cual lo principal no 
es la presencia-ausencia del diagnóstico clínico, sino la intensidad con la cual se presentan., ${ }^{44}$ La práctica de dietas y de ejercicio para bajar de peso, y la preocupación por tener una figura delgada, son normativas en la población y se presentan en una amplia gama dentro de la cual es difícil establecer un punto de corte entre lo normal y lo patológico. Para obtener una imagen más fina de la distribución de estas conductas en la población abierta, consideramos que sería necesario incrementar las posibilidades de la respuesta en términos de la frecuencia con la cual se realizan. Esto facilitaría el diferenciar entre sujetos sin preocupación por la delgadez, sujetos altamente preocupados, y casos de TCA, tal como ha sido propuesto por otros investigadores. ${ }^{35}$

En conclusión, los buenos resultados obtenidos nos permiten recomendar el uso de este instrumento como una alternativa breve, de fácil aplicación y calificación, que podría ser incluida en encuestas dirigidas a población abierta. Además de esta aproximación rápida y práctica para la obtención de datos sobre la prevalencia de conductas alimentarias de riesgo en nuestro país, el cuestionario puede facilitar la identificación temprana de casos y, con ello, propiciar la atención oportuna, con la finalidad de prevenir el desarrollo del trastorno.

Entre las limitaciones del estudio es importante mencionar que dentro de la muestra comunitaria no fue posible realizar una evaluación clínica diagnóstica que ayudara a descartar a las personas que pudieran tener el problema que se estudia. Por otro lado, los resultados deben ser tomados con cuidado, en virtud de que no se tiene una muestra representativa de la población mexicana y, como consecuencia, para el uso de este cuestionario en otras muestras, se recomienda probar su adecuado funcionamiento.

Aun con estas limitaciones, se considera que el trabajo presentado proporciona un acercamiento a la problemática de las conductas alimentarias de riesgo, tema que ha tenido escasa investigación en México.

\section{Referencias}

1. Guerro-Prado D, Barjau JM, Chinchilla A. La epidemiología de los trastornos alimentarios y la influencia de los medios masivos de comunicación: revisión de la literatura. Actas Esp Psiquiatr 2001; 29:403-410.

2. Kohn M, Golden N H. Eating disorders in children and adolescents: Epidemiology, diagnosis and treatment. Paediatr D rugs 2001;3:91-99. 3. American Psychiatric Association. Diagnostic and Statistical Manual of Mental Disorders DSM-IV. 4th edition.W ashington, DC:APA; 1994. 4. Shisslak CM, C rago M, Estes LS.The spectrum of eating disturbances. Int J Eat Disord 1995;18:209-219.
5. Mancilla JM,Alvarez G, López X, Mercado L, Manríquez E, Román M. Trastornos alimentarios y factores asociados en universitarias mexicanas. Psicol y Ciencia Soc 1998;2:34-58.

6. Gómez-Peresmitré G, Pineda G G, L'Esperance P, Hernández A, Platas $S$, León $R$. D ieta restrictiva y conducta alimentaria compulsiva en una muestra de adolescentes mexicanos. Rev Mex Psicol 2002;19:125-132. 7. Unikel C, Villatoro J, Medina-Mora ME,Alcantar EN , Fleiz C, Hernández SA. Conductas alimentarias de riesgo en adolescentes. Datos en población estudiantil del Distrito Federal. Rev Invest Clin 2000;52:140-147.

8. Strober M, Freeman R, Morell W. The long-term course of severe anorexia nervosa in adolescents: Survival analysis of recovery, relapse, and outcome predictors over 10-15 years in a prospective study. Int J Eat Disord 1997;22:339-360.

9. Herzog DB, Keller MB, Lavori PW . 0 utcome in anorexia nervosa and bulimia nervosa:A review of the literature.J N erv Ment $D$ is 1988;176:131-143.

10. N orring CEA, Sohlberg SS. 0 utcome, recovery, relapse and mortality across six years in patients with clinical eating disorders. Acta Psychatr Scand 1993;87:437.

11. Sullivan PF. Mortality in anorexia nervosa. Am J Psychiatry 1995;152:1073-1074.

12. Johnson JG, Cohen P, Kasen S, Brook JS. Eating disorders during adolescence and the risk for physical and mental disorders during early adulthood. Arch Gen Psychiatry 2002;59:545-552.

13. Brewerton TD, Lydiard RB, Herzog D B, Brotman AW, 0 'N eil PM, Ballenger JC. Comorbidity of axis I psychiatric disorders in bulimia nervosa. J Clin Psychiatry 1995;56:77-80.

14. Bulik CM, Sullivan PF, Carter FA, Joyce PR. Lifetime anxiety disorders in women with bulimia nervosa. Compr Psychiatry 1996;37:368-374.

15. Favaro A, Santonastaso P. Suicidality in eating disorders: C linical and psychological correlates. Acta Psychiatr Scand 1997;95:508-514. 16. Fava M,A braham M, Clancy-Colecchi K, Pava JA, Matthews J, Rosenbaum JF. Eating disorder symptomatology in major depression. J N erv Ment D is 1997;185:140-144.

17. Garner DM, Garfinkel PE.The Eating Attitudes Test:An index of the symptoms of anorexia nervosa. Psychol Med 1979;9:273-279.

18. Garner D M, O Imsted M, Polivy J. D evelopment and validation of a multidimensional eating disorder inventory for anorexia nervosa and bulimia. Int J Eat Disord 1983;2:15-33.

19. Alvarez G, Mancilla JM,Vázquez R, U nikel C, Caballero A, Mercado D. Validity of the Eating Attitudes Test:A study of Mexican eating disorders patients. J Eat W eight Disord Studies on anorexia bulimia obesity. En prensa.

20. Alvarez G. Evaluación de las propiedades psicométricas del Test de Actitudes A limentarias en mujeres mexicanas. Rev Mex de Psicol 2002:9:47-56.

21. Alvarez D, Franco K. Validación del Eating Disorders Inventory (EDI) en población mexicana (tesis). México, DF: Universidad N acional Autónoma de México, Campus-Iztacala; 2001.

22. Eddy KT, Hennessey M. Eating pathology in east African women:The role of media and globalization. A cad for Eat Disord N ewslett 2004;12:13.

23. Bojórquez I. Construcción de género, actitudes y conductas alimentarias en mujeres adolescentes mexicanas (tesis). México, D F: Universidad N acional Autónoma de México; 2004.

24. Unikel C. Factores de riesgo en los trastornos de la conducta alimentaria (tesis). México, D F: U niversidad $\mathrm{N}$ acional Autónoma de México; 2003

25. Unikel C, Gómez Peresmitré G.Validez de constructo de un instrumento para la detección de factores de riesgo en los trastornos de la conducta alimentaria en mujeres mexicanas. Salud Ment 2004;27:38-49. 
26. Radloff L.The CES-D Scale:A self-report depression scale for research in the general population.Appl Psychol Meas 1977;1:385-401. 27. Roberts E.The reliability of the CES-D Scale in different ethnic contexts. Psychiatry Res 1980;2:125-134.

28. González-Forteza C. Estrés psicosocial y respuestas de enfrentamiento: impacto sobre el estado emocional en adolescentes (tesis). México, DF: Universidad N acional Autónoma de México; 1992. 29. Mariño MC, Medina-Mora ME, C haparro JJ, G onzález C. Confiabilidad y estructura factorial del CES-D en adolescentes mexicanos. Rev Mex Psicol 1993;10:141-145

30. Harris EC, Barraclough B. Excess mortality of mental disorder. Br Psychiatry 1998; 173:11-53.

31. Corcos M,Taieb O, Benoit-Lamy S, Paternity S, Jeammet P, Flament MF. Suicide attempts in women with bulimia nervosa: Frequency and characteristics. Acta Psychiatr Scand 2002;106:381-386.
32. D awson B,Trapp R. Bioestadística médica. Métodos de medicina basada en evidencias. 3a edición. México, DF: El Manual Moderno; 2002:289-309

33. Altman D G, Bland JM. Statistics notes: Diagnostic tests 1: Sensitivity and specificity. BMJ 1994;308:1552.

34. Striegel-Moore RH. Prevention of bulimia nervosa: $Q$ uestions and challenges. En: Crowther H, Tennebaum DL, Hobfell SE, Stephens MA, ed.The etiology of bulimia nervosa:The individual and familial context. W ashington, DC: Hemisphere;1992:203-223.

35. W ood A, W aller G, G owers S. Predictors of eating psychopathology in adolescent girls. Eur Eat Disord Rev 1994;2(1):6-13

\section{A nexo}

\section{Cuestionario de conductas alimentarias de Riesgo}

En los últimos tres meses (marca una opción para cada inciso)

\begin{tabular}{|c|c|c|c|c|c|}
\hline & & $\begin{array}{c}\text { Nunca } \\
0 \text { casi nunca }\end{array}$ & A veces & $\begin{array}{c}\text { Con frecuencia } \\
\text { Dos veces en una semana }\end{array}$ & \begin{tabular}{|c|} 
Con mucha frecuencia \\
Más de dos veces en una semana
\end{tabular} \\
\hline 1 & Me ha preocupado engordar & & & & \\
\hline 2 & En ocasiones he comido demasiado, me he atascado de comida & & & & \\
\hline 3 & $\begin{array}{l}\text { He perdido el control sobre lo que como (tengo la sensación } \\
\text { de no poder parar de comer) }\end{array}$ & & & & \\
\hline 4 & He vomitado después de comer, para tratar de bajar de peso & & & & \\
\hline 5 & $\begin{array}{l}\text { He hecho ayunos (dejar de comer por } 12 \text { horas o más) } \\
\text { para tratar de bajar de peso }\end{array}$ & & & & \\
\hline 6 & He hecho dietas para tratar de bajar de peso & & & & \\
\hline 7 & He hecho ejercicio para tratar de bajar de peso & & & & \\
\hline 8 & $\begin{array}{l}\text { He usado pastillas para tratar de bajar de peso } \\
\text { Especifica cuál(es) }\end{array}$ & & & & \\
\hline 9 & $\begin{array}{l}\text { He tomado diuréticos (sustancia para perder agua) } \\
\text { para tratar de bajar de peso } \\
\text { Especifica cuál(es) }\end{array}$ & & & & \\
\hline 10 & $\begin{array}{l}\text { He tomado laxantes (sustancia para facilitar la evacuación) } \\
\text { para tratar de bajar de peso } \\
\text { Especifica cuál(es) }\end{array}$ & & & & \\
\hline
\end{tabular}

